\title{
Expectant Management Before In vitro Fertilization in Women Aged 39 or Above and Unexplained Infertility Does Not Decrease Live Birth Rates Compared to Immediate Treatment
}

\author{
Andrea Roberto Carosso ${ }^{1}$ (D) Rik van Eekelen ${ }^{2} \cdot$ Alberto Revelli $^{1} \cdot$ Stefano Canosa $^{1} \cdot$ Noemi Mercaldo $^{1} \cdot$ Ilaria Stura $^{3}$. \\ Stefano Cosma ${ }^{1}$. Carlotta Scarafia ${ }^{1} \cdot$ Chiara Benedetto $^{1}$. Gianluca Gennarelli ${ }^{1}$
}

Received: 18 June 2021 / Accepted: 5 October 2021 / Published online: 1 November 2021

(c) Society for Reproductive Investigation 2021

\begin{abstract}
Unexplained infertile couples can have further expectant management before starting assisted reproductive treatments. However, ovarian reserve and in vitro fertilization (IVF) outcomes rapidly decline after 39 years or more. It is thus important to clarify whether a waiting policy is also appropriate for women of advanced age. Couples who had access to a waiting list for approximately 1 year before receiving reimbursed public IVF were compared with those paying for access to immediate treatment. To allow for comparisons between these two strategies, we followed up couples who opted to pay for 1 year after the last embryo transfer from their first cycle. We calculated the proportion of live births in both groups and compared these using logistic regression models and a two-sample $\mathrm{Z}$ test for equality of proportions. Six hundred thirty-five couples were evaluated. Out of 359 couples in the immediate group, $70(19.5 \%)$ had a live birth of which 11 after natural conception and 59 after IVF. Out of 276 couples in the waiting group, 57 (20.7\%) had a live birth of which 37 after natural conception and 20 after IVF. There was no statistically significant difference between the two strategies in terms of the crude cumulative live birth rate (cLBR). The adjusted odds ratio of 0.69 (95\% CI:0.39-1.22) did not change this conclusion as our sensitivity analyses. The cLBR for the 'waiting before IVF' and the 'immediate' strategies were similar. Further studies are needed to better characterize couples affected by unexplained infertility in order to individualize treatment strategies.
\end{abstract}

Keywords IVF · Unexplained infertility $\cdot$ Natural conception $\cdot$ Expectant management $\cdot$ Individual prognoses $\cdot$ Advanced age

Andrea Roberto Carosso and Rik van Eekelen joint first authors.

Chiara Benedetto and Gianluca Gennarelli joint last authors.

Andrea Roberto Carosso

andrea88.carosso@gmail.com

1 Obstetrics and Gynecology 1U, Physiopathology of Reproduction and IVF Unit, Department of Surgical Sciences, Sant' Anna Hospital, University of Turin, Via Ventimiglia 1, 10126 Turin, Italy

2 Centre for Reproductive Medicine, Amsterdam UMC, Academic Medical Centre, Meibergdreef 9, 1105 AZ Amsterdam, the Netherlands

3 Department of Public Health and Pediatric Sciences, University of Turin, Turin, Italy

\section{Introduction}

A diagnosis of unexplained infertility is considered in couples who have tried to conceive for at least 1 year without success, despite clinical evidence of ovulation, tubal patency, and normal semen parameters [1]. Unexplained infertility accounts for approximately $30 \%$ of infertility in couples seeking in vitro fertilization (IVF) [2], though up to $50 \%$ with a diagnosis of unexplained infertility may conceive spontaneously after initial assessment [3].

Unexplained infertility comprises a heterogeneous group of conditions $[4,5]$, including the gradual decrease in fecundity with advancing age (age-related infertility) [6]. In women aged 40 years or more, the cumulative live birth rate after IVF is reportedly around 7\% [7]. Given the higher likelihood of unsuccessful IVF outcome, immediate IVF is recommended, particularly in women over 40 years $[8,9]$ in which there is a rapid decline in oocyte quantity 
and quality [10]. But how best to treat unexplained infertility in older women poses several challenges. A potential overuse of infertility treatments carries unnecessary health risks for the patient and incurs wastage of resources [11-13]. Whether reproductive aged women with unexplained infertility might benefit from immediate IVF treatment or from delayed treatment in the hope of achieving spontaneous conception remains an open question [14].

Previous studies that indirectly sought to address this issue reported higher pregnancy rates in older women with unexplained infertility after immediate IVF than after intrauterine insemination (IUI) [15]; nonetheless, a therapeutic space for IUI remains [16]. A recent meta-analysis found higher pregnancy rates after immediate IVF, although the quality of evidence was moderate and based on outcomes in 725 couples with a poor prognosis of natural conception [17].

Couples are increasingly concerned about access to IVF treatment since the outbreak of the COVID-19 pandemic [18], which prompted discontinuation of treatment due to limited knowledge about the potential effects of SARS$\mathrm{CoV}-2$ infection on pregnancy [19-22]. A silent cause of distress is the uncertainty surrounding the possible consequences of prolonged waiting for treatment outcomes [23, 24]. Just how long a couple with unexplained infertility and advanced woman's age can wait before starting IVF treatment is unknown. Some experts claim to wait a maximum of 6 months before accessing IVF treatment, but there is no strong evidence on how much further waiting can harm the couple in terms of IVF outcomes. As in other countries, couples entitled to assisted reproductive technology (ART) treatment covered by the Italian public health care system are put on a waiting list. The alternative option is to pay for treatment out of pocket. With the above points in mind, we compared live birth rates after delayed management versus immediate IVF treatment in couples with unexplained infertility and women aged 39 or older. To do this, we retrospectively retrieved data from a cohort of women who had waited 1 year before starting IVF treatment and from a cohort that had undergone immediate self-paid IVF treatment.

\section{Methods}

\section{Patient Selection and Follow-up}

The study was performed in accordance with the Helsinki Declaration and with approval of the City of Health and Science Institutional Review Board (n. 0,040,486). The informed consent of the patients to the treatment and data collection was obtained according to the indications of the City of Health and Science Institutional Review Board.
In this retrospective analysis, we included a cohort of women aged 39-43 years seeking infertility diagnosis and treatments, who were admitted to the IVF unit of the S. Anna academic hospital and to its private appendix between 2010 and 2019. Couples who enter a waiting list for publicly funded IVF and those receiving immediate self-paid treatment were compared. In both cases, the same diagnostic and therapeutic protocols were applied.

Only couples with unexplained infertility were selected. In detail, all women included in the study had ovulatory cycles and patency of at least one fallopian tube at hysterosonosalpingography (HSSG), whereas all male partners had normal basic semen parameters according to the indications of WHO 2010. Exclusion criteria were any known cause of female infertility (i.e. previous history of pelvic inflammatory disease, positive anti-Chlamydia IgG, endometriosis, anovulation, etc.), early follicular phase follicle stimulating hormone $(\mathrm{FSH})>20 \mathrm{UI} / \mathrm{l}$ and/or anti-Mullerian hormone $(\mathrm{AMH})<0.1 \mathrm{ng} / \mathrm{ml}$ and female body mass index $(\mathrm{BMI})>32 \mathrm{~kg} / \mathrm{m}^{2}$. A previous diagnosis of unexplained infertility at other IVF centres was not considered complete if these criteria were not met.

Couples were considered as part of the 'waiting' group if they abided by national protocol to wait for about 1 year after diagnosis, before IVF treatment. Couples were considered as part of the 'immediate' group if they opted to pay for access to immediate private IVF. We will also refer to these groups as 'strategies'. 'IVF cycle' was defined as the completion of all transfers deriving from the same ovarian stimulation and oocyte retrieval. Follow-up for the waiting group was up to their first IVF cycle. Follow-up for the immediate group was 1 year after their last embryo transfer from their first IVF cycle ('last embryo transfer' defines the last transfer of cryopreserved embryos obtained from the same IVF cycle). In the immediate strategy, some couples went through multiple cycles in the follow-up period. Only the first IVF cycle was analysed in order to allow follow-up for both groups to be similar. Figure 1 shows the timeline of 12 months plus 1 IVF cycle in total for both groups.

\section{IVF Procedure and Clinical Outcomes}

Controlled ovarian stimulation (COS) was performed either with recombinant FSH (rFSH), human

Menopausal Gonadotropin (HP-hMG) or rFSH plus recombinant luteinizing hormone $(\mathrm{rLH})$, under

pituitary suppression. The choice of the starting gonadotropin dose was based on age, BMI, antral

follicular count (AFC), AMH circulating concentrations, as well as on the response to previous COS. In the absence of any pre-fixed criteria, the COS regimen (type of protocol and type of medication) was decided and prescribed by 


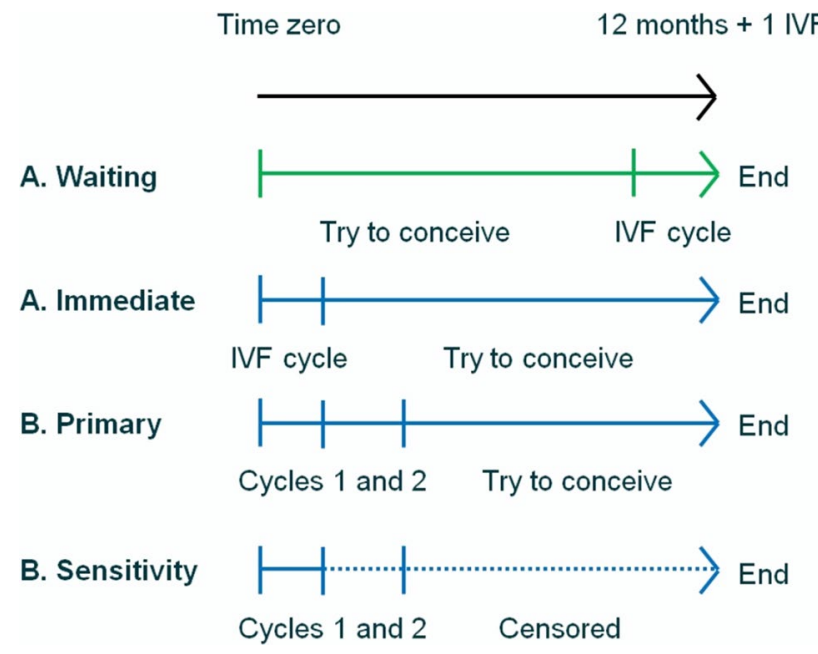

Fig. 1 Timeline of 12 months plus one cycle of IVF in the two groups (A). In (B), we show the two analyses for 473 couples in the immediate group who have received more than one IVF cycle: we either follow them between and 474 after unsuccessful IVF (primary analysis) or censor them after their 1st cycle (sensitivity)

different physicians of the IVF unit, according to their own clinical experience, as per real-life clinical practice.

Both long (Gn-RH agonist for pituitary suppression) and short (Gn-RH antagonist for pituitary suppression) protocols were used. COS was monitored by serial transvaginal US and serum estradiol (E2) measurements performed every second day from stimulation days 6-7. COS continued until at least two follicles reached $18 \mathrm{~mm}$ in mean diameter, when ovulation was triggered by injecting subcutaneously either 10,000 international units (IU) of hCG (Gonasi HP, IBSA, Lugano, Switzerland) or $250 \mathrm{mcg}$ of rHCG (Ovitrelle, Merck, Darmstadt, Germany). US-guided transvaginal oocyte pick-up (OPU) was performed approximately 36-37 h after hCG administration, under local anesthesia (paracervical block). Conventional IVF or ICSI was performed on all available oocytes within $4 \mathrm{~h}$ after oocyte collection or after $2 \mathrm{~h}$ from cumulus cell removal, respectively. Normal fertilization was assessed for the presence of two pronuclei (2PN) and the extrusion of the second polar body after $16-18 \mathrm{~h}$ post-insemination.

All cleaved embryos were morphologically evaluated under a conventional stereomicroscope, using the IMCS score by Holte [25]. According to the policy of our IVF unit during the time period under study, embryo transfer (ET) was performed either on day 2 or day 3 based on the presence of 1-3 embryos or $\geq 4$ embryos, respectively. Embryos were selected and transferred in uterus using a soft catheter, under ultrasound guidance, applying the method previously published by our group [26]. The remaining embryos (if any) were kept in culture until day 6 of development for cryopreservation at the blastocyst stage or discharge. The luteal phase was supported by administering $180 \mathrm{mg} /$ day natural progesterone (Crinone 8, Merck, Darmstadt, Germany) for 15 days. Pregnancy was assessed by serum hCG assay after 14 days from embryo transfer and then confirmed if at least one gestational sac was visualized on transvaginal US after 2 further weeks.

The primary outcome was the cumulative live birth rate (cLBR). The cLBR was defined as live deliveries (at least one live birth) per women over the full follow-up period. The full follow-up period was defined as either a period of expectant management up to the first IVF/ICSI cycle (including both fresh and frozen/thawed embryo transfers) or 1 year after the last transfer (fresh or frozen/thawed) of the first IVF/ICSI cycle. Live birth was defined as the delivery of a live-born infant ( $>24$ weeks of gestation).

\section{Analyses}

We tabulated the baseline patient characteristics for the two strategies separately.

As a crude analysis, we simply calculated the proportion of live births in both groups and compared these using a two-sample $\mathrm{Z}$ test for equality of proportions. We then calculated the crude odds ratio for immediate IVF versus waiting using a logistic regression model.

Next, we adjusted for confounding characteristics in the logistic regression model. In our study design, the only difference between strategies was the timing of IVF. However, as there was no random allocation to the waiting and immediate strategy, these groups could still differ in terms of prognostic characteristics associated with opting for waiting or immediate IVF. We had to adjust for these confounding characteristics that explained both the chance of live birth and the choice between waiting or immediate IVF. We identified the following characteristics a priori as confounders: female age, duration of infertility, primary or secondary infertility, previous miscarriage, previous IUI/IVF treatment, AMH, $\mathrm{AFC}$ and previous induced abortion.

\section{Sensitivity Analyses}

We also considered a more technical, complicated statistical analysis: the idea is to emulate a randomized controlled trial [27]. Rather than a simple adjustment in the logistic regression model, which is not strictly the same as a randomized trial, we conducted inverse probability weighting that weights some couples more than others to obtain a balance between groups [28]. We regressed the previously identified patient characteristics (female age, duration of infertility, primary or secondary infertility, previous miscarriage, previous IUI/IVF treatment, AMH, AFC and previous induced abortion) on the strategy groups i.e. 'immediate group (a ' 1 ') versus the waiting group (a ' 0 ')' using a logistic regression 
model. This model was used to predict the probability of receiving immediate IVF or waiting given a couples' individual characteristics. For couples who received immediate IVF, we divided the proportion of couples who received immediate IVF by a couple's individual probability of choosing immediate IVF [28]. For couples who waited, we divided the proportion of couples who waited by a couple's individual probability of waiting. The result was an individual weight value. This weight value counts certain couples more often than others in order to balance the cohort in terms of the mentioned baseline characteristics. This process was repeated until balance was found, defined as standardized mean differences below 0.1 .

The standard errors obtained from standard software are incorrect as they ignore the weighting procedure [28]. To obtain valid standard errors, we resampled 5000 times, repeated the procedure, calculated the statistic and reported their 2.5th and 97.5th percentile as the bootstrap results.

After we derived the weights, we first checked with a new tabulation whether the patient characteristics were similar between weighted groups, then repeated the previously conducted analyses in the weighted data: we calculated the proportion of live births in both weighted groups and compared these using a two-sample $\mathrm{Z}$ test for equality of proportions. In addition, we calculated the odds ratio for immediate IVF versus waiting using a logistic regression model including the weights.

Couples who opted to pay for immediate IVF could receive multiple IVF cycles, which leads to follow-up timelines that are slightly different. In the previous analyses, we assumed that these couples could still conceive naturally between IVF cycles and after their last IVF cycle. To assess the influence on this assumption on our results, we now considered couples who received more than one IVF cycle as censored, i.e. no longer pursuing expectant management after their first unsuccessful IVF cycle. After censoring, we estimated the proportion of live birth as the cumulative probability over one IVF cycle and 1 year of expectant management using the Kaplan-Meier method. Finally, we compared proportions between groups with $\mathrm{Z}$ tests as before.

\section{Software}

Data were prepared in Microsoft Excel. All analyses were conducted in $\mathrm{R}$ version 3.6.0 and RStudio ( $\mathrm{R}$ Core Team, 2013) using the rms, mice, dplyr and CreateTableOne packages.

\section{Results}

Data on 635 couples were available. Out of 359 couples in the immediate group, 70 (19.5\%) had a live birth of which 11 after natural conception and 59 after IVF. Out of 276 couples in the waiting group, $57(20.7 \%)$ had a live birth of which 37 after natural conception and 20 after IVF. Couples in the waiting group started their first IVF cycle after a median of 12 months (25th-75th percentile: 11-14).

Characteristics in both groups are depicted in Table 1.

There was no statistically significant difference between the two groups in terms of the cLBR (19.5\% immediate versus $20.7 \%$ waiting, $95 \% \mathrm{CI}$ for difference: -0.07 to 0.05 ). In addition, the crude odds ratio (OR) did not significantly differ from 1 (OR 0.93, 95\%CI: 0.63-1.38).

The adjusted OR for the immediate group compared to the waiting group was 0.69 (95\%CI: $0.39-1.22)$ and thus also not significant. In Table 2, we present the ORs for all characteristics.

\section{Sensitivity Analyses}

After weighting, there was an adequate balance between groups in terms of female age, duration of infertility, AMH, AFC, primary or secondary infertility, previous miscarriage, previous IUI/IVF treatment and previous induced abortion as shown by the point estimates provided in the Table 3 .

In the weighted cohort, there was no significant difference between the two groups in terms of the cLBR $(16.8 \%$ immediate versus $26.6 \%$ waiting, bootstrap 95\% CI for difference: -0.20 to 0.01 ). In addition, the OR did not significantly differ from 1 (OR 0.56 , bootstrap 95\% CI: 0.32-1.08).

In the sensitivity analysis in which we censored couples in the immediate group after their first unsuccessful IVF cycle if they received at least one addition cycle, the cLBR was $20.8 \%$ for the immediate group instead of $19.5 \%$. There was no statistically significant difference between the two groups in terms of the cLBR $(20.8 \%$ immediate versus $20.7 \%$ waiting, $95 \% \mathrm{CI}$ for difference: -0.07 to 0.06 ). In the weighted cohort, there was no significant difference between the two groups in terms of the cLBR (17.8\% versus $26.6 \%$, bootstrap $95 \% \mathrm{CI}$ for difference: -0.20 to 0.03 ).

\section{Discussion}

The main finding of this retrospective study involving couples with unexplained infertility and woman's age 39-43 years was that the cumulative 1-year live birth rate after one IVF cycle was comparable between the patients who had received delayed and immediate IVF treatment.

Recent studies involving large cohorts of infertile couples with varying woman's age have reported that the probability of achieving pregnancy is higher after IVF than after expectant management; however, the chances decrease with advancing woman's age [9]. The overall effect of increasing female age (per year) was also tested in our study, and we 
Table 1 Baseline characteristics of couples in the immediate or waiting group

\begin{tabular}{|c|c|c|c|}
\hline Baseline characteristics of couples & $\begin{array}{l}\text { Couples who received immediate } \\
\text { IVF }(n=359)\end{array}$ & $\begin{array}{l}\text { Couples who waited before IVF } \\
(n=276)\end{array}$ & $P$ value \\
\hline Female age at diagnosis (years) & $40.6(39.0-42.0)$ & $40.2(39.0-42.0)$ & $<0.05$ \\
\hline Male age at diagnosis (years) & $42.1(34.0-53.1)$ & $41.7(31.9-52.0)$ & 0.39 \\
\hline Duration of infertility at diagnosis (years, median) & $2.0(1.0-8.0)$ & $3.0(1.0-12.1)$ & $<0.05$ \\
\hline Percentage of progressive motile sperm (median) & $40.0(30.0-48.0)$ & $38.5(32.0-49.1)$ & $<0.05$ \\
\hline Total progressive sperm count $\left(10^{6}\right)$ (median) & $149(43-452)$ & $106(46-365)$ & $<0.05$ \\
\hline Female smoking status (yes versus no) & $44(12 \%)$ & $31(11 \%)$ & 0.78 \\
\hline $\mathrm{AFC}$ & $11.2(3.0-28.0)$ & $9.5(2.0-28.2)$ & $<0.05$ \\
\hline AMH (ng/ml, median) & $1.1(0.1-5.8)$ & $1.1(0.1-8.0)$ & 0.90 \\
\hline FSH (IU) & $8.9(3.9-17.9)$ & $8.4(3.7-16.6)$ & 0.12 \\
\hline Primary infertility (versus secondary) & $260(72 \%)$ & $116(42 \%)$ & $<0.05$ \\
\hline Previous miscarriage: & & & $<0.05$ \\
\hline None & $294(82 \%)$ & $153(55 \%)$ & \\
\hline 1 & $49(14 \%)$ & $78(28 \%)$ & \\
\hline 2 & $11(3 \%)$ & $27(11 \%)$ & \\
\hline 3 or more & $5(1 \%)$ & $18(7 \%)$ & \\
\hline Received previous ART & & & $<0.05$ \\
\hline None & $234(65 \%)$ & $43(16 \%)$ & \\
\hline IUI & $18(5 \%)$ & $190(69 \%)$ & \\
\hline IVF & $107(30 \%)$ & $43(16 \%)$ & \\
\hline $\begin{array}{l}\text { Previous induced abortion } \\
\text { (yes versus no) }\end{array}$ & $14(4 \%)$ & $31(11 \%)$ & $<0.05$ \\
\hline ICSI (instead of IVF) & $163(45 \%)$ & $128(46 \%)$ & 0.81 \\
\hline Starting dose of FSH & $300(225-300)$ & $283.5(150-300)$ & 0.25 \\
\hline \multicolumn{4}{|l|}{ Drug: } \\
\hline FSH & $100(28 \%)$ & $72(26 \%)$ & \\
\hline $\mathrm{FSH}+\mathrm{LH}$ & $64(18 \%)$ & $82(30 \%)$ & \\
\hline hMG & $195(54 \%)$ & $122(44 \%)$ & \\
\hline
\end{tabular}

Data are mean unless specified as median. Between brackets depicts the percentage for categorical data or the 5th-95th percentile for continuous data. AFC antral follicle count, $A M H$ anti-Müllerian hormone; $F S H$ follicle stimulating hormone

found this to be non-significant associated to the cumulative live birth rate (Table 2): this somewhat surprising result can be explained in two ways. First, the range of female age is relatively narrow in our population (39 to 43 ), so there are not many between-couple comparisons to be drawn. In addition, the effect of female age here represents a combination of age on natural conception and age of conception after IVF, which is difficult to interpret [9]. This raises the question of how long we can delay treatment without causing the patient harm and allow time for the couple to conceive spontaneously. According to a recent retrospective study, a delay of up to 6 months is not associated with poor IVF outcomes in patients with reduced ovarian reserve [29]. But because the chances of achieving pregnancy without IVF were not investigated, the dilemma remains to be solved.

Treatment strategies for reproductive aged women have gained greater interest with the shift to later childbearing $[30,31]$. Recommendations by medical societies suggest rapid diagnosis starting at age 35 years; however, consensus is lacking on the best treatment strategies when common causes of female and male infertility have been ruled out $[17,32,33]$. This uncertainty persists also in women categorized as poor responders by commonly shared criteria [34]. Though such patients should receive IVF treatment as soon as possible, it may be too early to establish whether the infertility is due to reduced ovarian reserve or perhaps to other unexplained factors. The idea that IVF can overcome age-related subfertility in women is doubtful: IVF does not enhance oocyte quality, and ovarian responsiveness decreases with time, raising the risk of poor response to ovarian stimulation [35-37].

Our findings indicate that an expectant management strategy before IVF need not be considered ineffective or potentially detrimental; we found no evidence for a negative effect on live birth rate for the women in the delayed treatment group. The two cohorts included women aged 
Table 2 Adjusted odds ratios in the logistic regression model on the outcome live birth. This was the extended model, including adjustments

\begin{tabular}{ll}
\hline Baseline characteristics & Odds ratio (95\%CI) \\
\hline Scenario for immediate IVF (versus waiting) & $0.69(0.39-1.22)$ \\
Female age at diagnosis (per year) & $1.09(0.89-1.34)$ \\
Duration of infertility at diagnosis (per year) & $0.91(0.82-1.01)$ \\
AFC (per follicle) & $1.03(0.99-1.07)$ \\
AMH (per unit ng/ml) & $0.96(0.83-1.12)$ \\
Primary infertility (versus secondary) & $0.81(0.39-1.70)$ \\
Previous miscarriage: & \\
$\quad$ None & Reference \\
1 & $1.32(0.62-2.82)$ \\
2 & $0.87(0.30-2.49)$ \\
3 or more & $1.36(0.59-3.11)$ \\
Received previous ART & \\
None & Reference \\
IUI & $0.66(0.36-1.22)$ \\
IVF & $1.33(0.80-2.20)$ \\
Previous induced abortion (yes versus no) & $1.36(0.59-3.11)$ \\
\hline
\end{tabular}

Note: the logistic regression model was adjusted for female age, duration of infertility, AFC, AMH, primary infertility, previous miscarriage, previous ART and previous induced abortion

$A F C$ antral follicle count, $A M H$ anti-Müllerian hormone, $F S H$ follicle-stimulating hormone
39 or older with acceptable ovarian reserve, as measured by AFC and AMH levels: whether these results are reproducible also in women with severely diminished ovarian reserve is uncertain. While couples who have experienced or will experience delays in accessing IVF treatments due to the COVID-19 pandemic may find these findings reassuring [29], they should be advised that after age 39 neither delayed nor immediate IVF treatment increases the probability of pregnancy with a live birth. In brief, reproductive counselling is warranted in younger women wishing to delay motherhood.

Our study findings add to the current debate on the optimal therapeutic approach to unexplained infertility, as couples that conceive after IVF might have otherwise conceived naturally $[1,12,38]$. The $37(13 \%)$ couples in the delayed treatment group achieved spontaneous pregnancy with a live birth during the observation period. This rate was far lower compared to a previous study with a design similar to ours [39], but the inclusion of younger patients may explain the higher pregnancy rates. Similar to the rates we recorded, a Dutch study involving couples waiting for IVF reported a $14 \%$ cumulative live birth rate over 1 year [40]. Both of these early studies $[39,40]$ and a more recent larger one [9] found immediate IVF superior to expectant management also in couples with unexplained infertility. Since such couples can still conceive naturally, the question is not whether but
Table 3 Baseline characteristics of couples in the waiting or immediate group after weighting

\begin{tabular}{lll}
\hline Baseline characteristics & $\begin{array}{l}\text { Couples who received } \\
\text { immediate IVF }(n=344)\end{array}$ & $\begin{array}{l}\text { Couples who waited } \\
\text { before IVF }(n=291)\end{array}$ \\
\hline Female age at diagnosis (years) & 40.3 & 40.3 \\
Male age at diagnosis (years) & 41.6 & 41.7 \\
Duration of infertility at diagnosis (years, median) & 2 & 2 \\
Percentage of progressive motile sperm (median) & 42 & 36 \\
Total progressive sperm count $\left(10^{6}\right)$ & 152 & 109 \\
Female smoking status (yes versus no) & $54(16 \%)$ & $37(16 \%)$ \\
AFC & 10.8 & 10.8 \\
AMH (ng/ml, median) & 1.2 & 1.2 \\
FSH (IU) & 8.7 & 8.1 \\
Primary infertility (versus secondary) & $218(63 \%)$ & $184(63 \%)$ \\
Previous miscarriage: & & \\
None & $257(75 \%)$ & $216(75 \%)$ \\
1 & $63(18 \%)$ & $54(19 \%)$ \\
2 & $15(4 \%)$ & $12(4 \%)$ \\
3 or more & $9(3 \%)$ & $8(3 \%)$ \\
Received previous ART & & $125(43 \%)$ \\
None & $150(44 \%)$ & $79(27 \%)$ \\
IUI & $91(27 \%)$ & $86(30 \%)$ \\
IVF & $103(30 \%)$ & $17(6 \%)$ \\
Previous induced abortion (yes versus no) & $21(6 \%)$ &
\end{tabular}

Data are mean unless specified as median. AFC antral follicle count, $A M H$ anti-Müllerian hormone, $F S H$ follicle stimulating hormone 
when they should receive IVF. If treatment is delayed and the couples conceive naturally, they are spared potentially premature, expensive and invasive treatment [1, 41, 42] and the offspring are spared exposure to the potential risks associated with ART [43, 44].

Confirmation of the present findings in larger series of patients may necessitate revision of protocols for treating unexplained infertility in reproductive aged women. Waiting before treatment or selecting women who are expected to benefit most from IVF could save couples from unnecessary invasive treatments while optimizing allocation of economic resources [9, 13, 14, 18, 42].

A major strength of our study is that the patients in both cohorts were managed by the same medical and embryologist team that followed identical diagnostic and therapeutic protocols. Furthermore, couples in Italy have the option to wait or pay out of pocket for immediate IVF treatment. This condition cannot be easily accepted in a prospective randomized controlled trial (RCT). RCTs investigating unexplained infertility that include a no treatment arm (i.e. expectant management) are lacking since clinicians might be reluctant to include such an arm. Furthermore, couples may perceive further expectant management as a waste of time and may be unwilling to volunteer in a study in which they might be allocated to the expectant management arm, whereas they will receive IVF if they refuse to enter the study [45]. Likewise, couples informed of their chances of spontaneous conception may refuse to adhere to IVF treatment or to seek further diagnostic testing. So, it is not surprising that an RCT similar in design to our study was stopped due to a low adhesion rate (Trial NL5384NTR5484). Because of lack of evidence from RCTs, most studies published so far have compared treatment arms or separate observational databases [15, 46, 47].

A major limitation to our methodological approach is the potential selection bias associated with choosing either treatment strategy. Although the ovarian reserve was similar in both groups (see AFC and AMH levels in Table 1), they differed by several baseline characteristics known to influence chances of pregnancy: prevalence of primary/ secondary infertility, history of previous miscarriage, and previous IUI/IVF. By applying inverse probability weighting [28], we were able to reasonably account for these confounding variables after weighting (Table 2). Furthermore, the duration of infertility was often longer than the 12 months usually required for diagnosis. This reflects an underestimation of the infertility diagnosis, which is often overlooked by couples or insufficiently investigated by general practitioners. We cannot know whether such a long period of infertility impacted our analysis. Since there was no significant difference in this parameter between the two groups, the comparison can be assumed to be reliable.

The couples undergoing multiple IVF cycles in the immediate treatment group might have had follow-up timelines (time available for spontaneous conception) that were not strictly comparable with the other group. However, most of the couples in the immediate treatment group who received multiple IVF cycles were still at risk of natural conception for most of the year. This is likely, given that the majority of couples that received multiple cycles in the immediate treatment group did not conceive after their first IVF cycle and remained free for several months between cycles for potential spontaneous conception.

We believe our primary analysis is realistic. The sensitivity analysis showed an estimated (unadjusted) proportion of live births of $20.8 \%$ versus $19.5 \%$, which did not affect the study conclusions. We argue that, given the study design, this difference does not substantially influence the results. Finally, it is increasing common to perform a single blastocyst transfer also in older couples. A further limitation of our study is that fresh-transfer was performed with embryos on day $2 / 3$ and only those from the frozen to the blastocyst stage. In the absence of strong evidence for the one or the other strategy, we believe that our results can still be considered useful, given that many IVF treatment centres still work on day $2 / 3$ fresh-transfers.

In conclusion, our study showed similar cumulative live birth rates in couples with unexplained infertility and advanced woman's age that received delayed and immediate IVF treatment. We found no evidence of cause for concern in couples currently awaiting IVF treatment. This may be comforting for couples for which treatment has been or will be delayed due to the COVID-19 pandemic. Further studies are needed to confirm these findings and to optimize economic resources, particularly in a setting of publicly funded IVF treatment.

Authors' Contributions Andrea Carosso conceived the study and wrote the manuscript. Rik van Eekelen performed the data analysis and cowrote the manuscript. Alberto Revelli contributes to interpretation of the data and critically revised the manuscript. Stefano Canosa, Noemi Mercaldo, and Ilaria Stura collect the data and contribute to data analysis. Stefano Cosma and Carlotta Scarafia contributed to execution of the study and critically revised the manuscript. Chiara Benedetto and Gianluca Gennarelli contribute to conceive the study, coordinate the study, and critically revised the manuscript. All authors reviewed and approved the final version of the manuscript.

Data Availability All data are available under request to the authors.

"The pre-print version of this article is present on https://assets. researchsquare.com/files/rs-131936/v1/b0c1f6be-1c1c-42b7-aa1d4 aef650bb9c1.pdf. This article is not published nor is under publication elsewhere."

\section{Declarations}

Ethics Approval All procedures performed in studies involving human participants were in accordance with the ethical standards of the institutional and/or national research committee and with the 1964 Helsinki Declaration and its later amendments or comparable ethical standards. The study was approved by the Institutional Review Board of Città della Salute e della Scienza di Torino (authorization n. 0040486). 
Consent to Participate and for Publication Informed consent was obtained from all individual participants included in the study according to the Institutional Review Board indications.

Conflict of Interests The authors declare no competing interests.

\section{References}

1. Kamphuis EI, Bhattacharya S, van der Veen F, Mol BWJ, Templeton A. Evidence based IVF group. Are we overusing IVF? BMJ. 2014;348:g252.

2. Brandes M, Hamilton CJCM, de Bruin JP, Nelen WLDM, Kremer JAM. The relative contribution of IVF to the total ongoing pregnancy rate in a subfertile cohort. Hum Reprod. 2010;25:118-26.

3. Brandes M, van der Steen JOM, Bokdam SB, Hamilton CJCM, de Bruin JP, Nelen WLDM, et al. When and why do subfertile couples discontinue their fertility care? A longitudinal cohort study in a secondary care subfertility population. Hum Reprod. 2009;24:3127-35.

4. Somigliana E, Paffoni A, Busnelli A, Filippi F, Pagliardini L, Vigano P, et al. Age-related infertility and unexplained infertility: an intricate clinical dilemma. Hum Reprod. 2016;31:1390-6.

5. Siristatidis C, Bhattacharya S. Unexplained infertility: does it really exist? Does it matter? Hum Reprod Oxford Academic. 2007;22:2084-7.

6. ESHRE Capri Workshop Group. A prognosis-based approach to infertility: understanding the role of time. Hum Reprod. 2017;32:1556-9.

7. Gennarelli G, Carosso A, Canosa S, Filippini C, Cesarano S, Scarafia $\mathrm{C}$, et al. ICSI versus conventional IVF in women aged 40 Years or more and unexplained infertility: a retrospective evaluation of 685 cycles with propensity score model. J Clin Med. 2019;8(10):1694.

8. McLernon DJ, Steyerberg EW, Te Velde ER, Lee AJ, Bhattacharya $S$. Predicting the chances of a live birth after one or more complete cycles of in vitro fertilisation: population based study of linked cycle data from 113873 women. BMJ. 2016;355:i5735.

9. van Eekelen R, van Geloven N, van Wely M, Bhattacharya S, van der Veen F, Eijkemans MJ, et al. IVF for unexplained subfertility; whom should we treat? Hum Reprod. 2019;34:1249-59.

10. Iliodromiti S, Iglesias Sanchez C, Messow C-M, Cruz M, Garcia Velasco J, Nelson SM. Excessive age-related decline in functional ovarian reserve in infertile women: prospective cohort of 15,500 women. J Clin Endocrinol Metab. 2016;101:3548-54.

11. Mennini FS, Marcellusi A, Viti R, Bini C, Carosso A, Revelli A, et al. Probabilistic cost-effectiveness analysis of controlled ovarian stimulation with recombinant FSH plus recombinant LH vs. human menopausal gonadotropin for women undergoing IVF. Reprod Biol Endocrinol. 2018;16:68.

12. Tjon-Kon-Fat RI, Bensdorp AJ, Scholten I, Repping S, van Wely $\mathrm{M}, \mathrm{Mol} \mathrm{BWJ}$, et al. IUI and IVF for unexplained subfertility: where did we go wrong? Hum Reprod. 2016;31:2665-7.

13 Eijkemans MJC, Kersten FAM, Lintsen AME, Hunault CC, Bouwmans CAM, Roijen LH, et al. Cost-effectiveness of "immediate IVF" versus "delayed IVF": a prospective study. Hum Reprod. 2017;32:999-1008.

14. van Eekelen R, van Geloven N, van Wely M, McLernon DJ, Eijkemans MJ, Repping S, et al. Constructing the crystal ball: how to get reliable prognostic information for the management of subfertile couples. Hum Reprod. 2017;32:2153-8.
15 Goldman MB, Thornton KL, Ryley D, Alper MM, Fung JL, Hornstein $\mathrm{MD}$, et al. A randomized clinical trial to determine optimal infertility treatment in older couples: the Forty and Over Treatment Trial (FORT-T). Fertil Steril. 2014;101(1574-1581):e1-2.

16. Farquhar CM, Liu E, Armstrong S, Arroll N, Lensen S, Brown J. Intrauterine insemination with ovarian stimulation versus expectant management for unexplained infertility (TUI): a pragmatic, open-label, randomised, controlled, two-centre trial. Lancet. 2018;391:441-50.

17 Wang R, Danhof NA, Tjon-Kon-Fat RI, Eijkemans MJ, Bossuyt $\mathrm{PM}$, Mochtar MH, et al. Interventions for unexplained infertility: a systematic review and network meta-analysis. Cochrane Database Syst Rev. 2019;9:CD012692.

18. Bhattacharya S, Maheshwari A, Ratna MB, van Eekelen R, Mol BW, McLernon DJ. Prioritising IVF treatment in the post COVID 19 era: a predictive modelling study based on UK national data. Hum Reprod. 2020;36(3):666-75.

19. Cosma S, Carosso AR, Cusato J, Borella F, Carosso M, Bovetti M, et al. Coronavirus disease 2019 and first-trimester spontaneous abortion: a case-control study of 225 pregnant patients. Am J Obstet Gynecol. 2020;224(4):391.e1-391.e7.

20. Smith ADAC, Gromski PS, Rashid KA, Tilling K, Lawlor DA, Nelson SM. Population implications of cessation of IVF during the COVID19 pandemic. Reprod Biomed Online. 2020;41:428-30.

21. Carosso A, Cosma S, Borella F, Marozio L, Coscia A, Ghisetti V, et al. Pre-labor anorectal swab for SARS-CoV-2 in COVID-19 pregnant patients: is it time to think about it? Eur J Obstet Gynecol Reprod Biol. 2020;249:98-9.

22. Veiga A, Gianaroli L, Ory S, Horton M, Feinberg E, Penzias A. Assisted reproduction and COVID-19: a joint statement of ASRM, ESHRE and IFFS. Hum Reprod Open. Oxford Academic 2020;2020(3):hoaa033.

23. Ben-Kimhy R, Youngster M, Medina-Artom TR, Avraham S, Gat I, Haham LM, et al. Fertility patients under COVID-19: attitudes, perceptions, and psychological reactions. Hum Reprod. 2020;35(12):2774-83.

24. Esposito V, Rania E, Lico D, Pedri S, Fiorenza A, Strati MF, et al. Influence of COVID-19 pandemic on the psychological status of infertile couples. Eur J Obstet Gynecol Reprod Biol. 2020;253:148-53.

25. Holte J, Berglund L, Milton K, Garello C, Gennarelli G, Revelli A, et al. Construction of an evidence-based integrated morphology cleavage embryo score for implantation potential of embryos scored and transferred on day 2 after oocyte retrieval. Hum Reprod. 2007;22:548-57.

26. Revelli A, Gennarelli G, Biasoni V, Chiadò A, Carosso A, Evangelista $\mathrm{F}$, et al. The ovarian sensitivity index (OSI) significantly correlates with ovarian reserve biomarkers, is more predictive of clinical pregnancy than the total number of oocytes, and is consistent in consecutive IVF cycles. J Clin Med. 2020;9(6):1914.

27. Hernán MA, Robins JM. Using big data to emulate a target trial when a randomized trial is not available. Am J Epidemiol. 2016;183:758-64.

28. Cole SR, Hernán MA. Constructing inverse probability weights for marginal structural models. Am J Epidemiol. 2008;168:656-64.

29. Romanski PA, Bortoletto P, Rosenwaks Z, Schattman GL. Delay in IVF treatment up to 180 days does not affect pregnancy outcomes in women with diminished ovarian reserve. Hum Reprod (Oxford, England). 2020;35:1630-6.

30. Adamson GD, de Mouzon J, Chambers GM, Zegers-Hochschild $\mathrm{F}$, Mansour R, Ishihara $\mathrm{O}$, et al. International committee for monitoring assisted reproductive technology: world report on assisted reproductive technology, 2011. Fertil Steril. 2018;110:1067-80.

31. De Geyter C, Calhaz-Jorge C, Kupka MS, Wyns C, Mocanu E, Motrenko T, et al. ART in Europe, 2015: results 
generated from European registries by ESHRE. Hum Reprod Open. 2020;2020(1):hoz038.

32. National Collaborating Centre for Women's and Children's Health (UK). Fertility: Assessment and Treatment for People with Fertility Problems [Internet]. London: Royal College of Obstetricians \& Gynaecologists; 2013 [cited 2020 Mar 27]. Available from: http:// www.ncbi.nlm.nih.gov/books/NBK247932/.

33. Evidence-based treatments for couples with unexplained infertility. a guideline. Fertil Steril. 2020;113:305-22.

34. Ferraretti AP, La Marca A, Fauser BCJM, Tarlatzis B, Nargund G, Gianaroli L, et al. ESHRE consensus on the definition of "poor response" to ovarian stimulation for in vitro fertilization: the Bologna criteria. Hum Reprod. 2011;26:1616-24.

35. Drakopoulos P, Romito A, Errázuriz J, Santos-Ribeiro S, PopovicTodorovic B, Racca A, et al. Modified natural cycle IVF versus conventional stimulation in advanced-age Bologna poor responders. Reprod Biomed Online. 2019;39:698-703.

36 Polyzos NP, Corona R, Van De Vijver A, Blockeel C, Drakopoulos $\mathrm{P}$, Vloeberghs V, et al. Corifollitropin alfa followed by hpHMG in GnRH agonist protocols. Two prospective feasibility studies in poor ovarian responders. Gynecol Endocrinol. 2015;31:885-90.

37. Revelli A, Carosso A, Grassi G, Gennarelli G, Canosa S, Benedetto C. Empty follicle syndrome revisited: definition, incidence, aetiology, early diagnosis and treatment. Reprod Biomed Online. 2017;35:132-8.

38. Fauser BCJM. Therapy: Unexplained infertility - ongoing transatlantic debate. Nat Rev Endocrinol. 2016;12:8-10.

39. Donderwinkel PF, van der Vaart H, Wolters VM, Simons AH, Kroon G, Heineman MJ. Treatment of patients with long-standing unexplained subfertility with in vitro fertilization. Fertil Steril. 2000;73:334-7.
40 Eijkemans MJC, Lintsen AME, Hunault CC, Bouwmans CAM, Hakkaart L, Braat DDM, et al. Pregnancy chances on an IVF/ICSI waiting list: a national prospective cohort study. Hum Reprod. 2008;23:1627-32.

41. te Velde ER, Eijkemans R, Habbema HD. Variation in couple fecundity and time to pregnancy, an essential concept in human reproduction. Lancet. 2000;355:1928-9.

42. van Eekelen R, Eijkemans MJ, Mochtar M, Mol F, Mol BW, Groen $\mathrm{H}$, et al. Cost-effectiveness of medically assisted reproduction or expectant management for unexplained subfertility: when to start treatment? Hum Reprod. 2020;35(9):2037-46.

43. Chen M, Heilbronn LK. The health outcomes of human offspring conceived by assisted reproductive technologies (ART). J Dev Orig Health Dis. 2017;8:388-402.

44. La Rovere M, Franzago M, Stuppia L. Epigenetics and Neurological Disorders in ART. Int J Mol Sci. 2019;20(17):4169.

45. van den Boogaard NM, van den Boogaard E, Bokslag A, van Zwieten MCB, Hompes PGA, Bhattacharya S, et al. Patients' and professionals' barriers and facilitators of tailored expectant management in subfertile couples with a good prognosis of a natural conception. Hum Reprod. 2011;26:2122-8.

46. Tjon-Kon-Fat RI, Tajik P, Zafarmand MH, Bensdorp AJ, Bossuyt PMM, Oosterhuis GJE, et al. IVF or IUI as first-line treatment in unexplained subfertility: the conundrum of treatment selection markers. Hum Reprod. 2017;32:1028-32.

47. van Eekelen R, Tjon-Kon-Fat RI, Bossuyt PMM, van Geloven $\mathrm{N}$, Eijkemans MJC, Bensdorp AJ, et al. Natural conception rates in couples with unexplained or mild male subfertility scheduled for fertility treatment: a secondary analysis of a randomized controlled trial. Hum Reprod. 2018;33:919-23. 